\title{
A LARGE ANIMAL MODEL FOR A FAILED TWO-STAGE REVISION OF INTRAMEDULLARY NAIL-RELATED INFECTION BY METHICILLIN- RESISTANT STAPHYLOCOCCUS AUREUS
}

\author{
T.F. Moriarty ${ }^{1}$, T. Schmid ${ }^{1}$, V. Post ${ }^{1}$, E. Samara ${ }^{1}$, S. Kates ${ }^{2}$, E.M. Schwarz ${ }^{3}$, S. Zeiter ${ }^{1}$ and R.G. Richards ${ }^{1}$ \\ ${ }^{1} \mathrm{AO}$ Research Institute Davos, Davos, Switzerland \\ ${ }^{2}$ Virginia Commonwealth University School of Medicine, Richmond, VA, USAA \\ ${ }^{3}$ Centre for Musculoskeletal Research, University of Rochester Medical Centre, Rochester, NY, USA
}

\begin{abstract}
The treatment of chronic orthopaedic device-associated infection (ODRI) often requires multiple surgeries and prolonged antibiotic therapy. Despite this extensive treatment protocol, the procedure is associated with significant failure rates. Currently, no large animal model is available that recapitulates a failed revision. Therefore, our aim was to establish a large animal model for failed treatment of an ODRI, in order to serve as a testbed for future interventional strategies.

Adult Swiss Alpine sheep received an intramedullary nail in the tibia and a localised inoculum of either a methicillin-sensitive or methicillin-resistant Staphylococcus aureus (MSSA, MRSA respectively). After 8 weeks, when chronic infection had been established, the animals underwent a staged revision with debridement and temporary placement of an antibiotic-loaded cement spacer. Antibiotics were delivered systemically in a standard or pathogen-adapted manner.

Debridement and implant exchange alone failed to treat the MSSA infection. Neither local therapy alone nor systemic therapy alone were effective in resolving infection with MSSA, but a combination of local and systemic therapy was effective against it. MRSA infection was not resolved by the combination of local and systemic antibiotics (standard or pathogen-adapted).

A model for failed revision of MRSA infection is described despite the use of local and systemic antibiotics. Novel interventions may be assessed using this model, including antibiotic and non-antibiotic interventions.
\end{abstract}

Keywords: Staged revision, osteomyelitis, Staphylococcus aureus, intramedullary nail, MRSA, antibiotic-loaded bone cement, local antibiotic, systemic antibiotic, antibiotic cement nail.

*Address for correspondence: T.F. Moriarty, AO Research Institute Davos, Clavadelerstrasse 8, 7270 Davos Platz, Switzerland.

Telephone: +41814142397_Ｅmail: fintan.moriarty@aofoundation.org

\section{Introduction}

The incidence of orthopaedic device-related infection (ODRI) following elective orthopaedic surgery is in the range of $0.7 \%$ to $4.2 \%$ (Kurtz et al., 2008; Sugarman and Young, 1989; Zimmerli et al., 2004), while the incidence in non-elective trauma cases ranges from approximately $1 \%$, after operative fixation of closed low-energy fractures, to more than $30 \%$ in complex open tibia fractures (Boxma et al., 1996; Patzakis and Wilkins, 1989). Approximately $35 \%$ of these infections are caused by Staphylococcus aureus (S. aureus) (Trampuz and Zimmerli, 2006) and, of these, up to $70 \%$ may be caused by antibioticresistant strains (e.g. methicillin-resistant S. aureus, MRSA) (Morgenstern et al., 2016).

Treatment concepts for fracture-related infection (FRI) have evolved over the years, although the variability of traumatic fractures, and associated soft tissue damage, has resulted in a great diversity of treatments and empirical approaches for FRI, which contrasts with the more defined algorithms for periprosthetic joint infection (PJI) (Osmon et al., 2013; Zimmerli, 2014). One of the critical parameters in determining the treatment strategy for ODRI is the time elapsed following initial surgery. Early infections (i.e. those occurring within 2 weeks from the primary surgery) may be treated by debridement, device retention and systemic antibiotics. However, infections diagnosed after this time (i.e. delayed or late infections) are more often treated with debridement and device removal or exchange (Lichstein et al., 2014; Strange et al., 2016). Device removal is required in these cases in order to remove the biofilm that may have formed upon it, which would otherwise resist antibiotic therapy and result in treatment failure. Device removal in FRI often involves external fixation, although a two-stage procedure (staged 
re-nailing) with an intra-stage antibiotic-loaded cement nail was also described (Wasko and Borens, 2013; Wasko and Kaminski, 2015). Despite extensive treatment protocols involving at least two surgeries, current estimates indicate that reinfection rates are significant. The average failure rate of two-stage exchange in PJI is $18 \%$, although even higher failure rates have been documented in certain situations (Haleem et al., 2004; Mortazavi et al., 2011; Pelt et al., 2014; Sherrell et al., 2011). For example, very poor outcomes are observed in patients with a prior failed debridement and exchange procedure [reinfection rate of $34 \%$ (Sherrell et al., 2011)], those infected with antibiotic-resistant pathogens [e.g. MRSA reinfection rate of $>42 \%$ (Kilgus et al., 2002; Mortazavi et al., 2011)] or patients with culture-negative infection (Mortazavi et al., 2011). Equivalent large-scale data are not available for FRI treated with staged renailing. The reports that are available describe small populations, few resistant pathogens and indicate that multiple revision surgeries are frequently required (Wasko and Borens, 2013). The high rates of treatment failure suggest that the pathogen, particularly true in the case of MRSA infection, is not eradicated by local and systemic antibiotic therapy and that the host immune defences are unable to prevent regrowth and colonisation of the new implant.

Any improvement in the success rate of the treatment of these infections may require a more complete understanding of why treatment fails. Preclinical in vivo models offer the possibility of reproducible, controlled conditions for studying treatment failure. Whilst a range of animal models of device-related bone infection have been described in the literature (see reviews: Calabro et al., 2013; Reizner et al., 2014), there are comparatively few models involving the treatment of device infection (e.g. Brady et al., 2011; Gatin et al., 2014; Inzana et al., 2015; Moskowitz et al., 2010). Furthermore, there are no models of staged hardware exchange due to infection available, to the best of our knowledge.

The aim of the present study was to develop a large animal model of a staged exchange following infection associated with an intramedullary nail. In order to minimise the burden upon the animals and minimise the risk of loss of animals due to surgical complications, a non-fracture model was used. Our primary aim was to provide a model for a failed revision despite surgical debridement, device exchange and local and systemic antibiotic administration. This model may then serve as a baseline against which new interventions may be compared.

\section{Materials and Methods}

\section{Institutional animal care and use committee (IACUC) approval and animal welfare}

Approval to perform this study was granted by the ethical committee of the canton of Grisons in Switzerland (approval numbers 34_2013 and 39_2014). All procedures were performed in an Association for Assessment and Accreditation of Laboratory Animal Care (AAALAC) International approved facility and according to Swiss animal protection law and regulations. 33 skeletally mature ( 2 to 4 years old), female Swiss Alpine sheep, with a weight range of $54-91 \mathrm{~kg}$ (average $74.9 \mathrm{~kg}$ ), were included in this study. Prior to inclusion in the study, each sheep underwent a complete physical examination including complete blood count (CBC) and a radiographic screening to ensure the tibia could accommodate the intramedullary (IM) nail. The sheep were group-housed for at least 2 weeks prior to surgery to acclimatise to housing conditions (daily cycles of $12 \mathrm{~h}$ light/dark) and were fed twice per day with hay, a mineral lick, and hand-fed grain to gain familiarity with caregivers.

\section{Study design and treatment overview}

The establishment of device-related bone infection, and subsequent medical and surgical treatment, required three surgeries per sheep over a total of 14 weeks. A schematic overview of the surgical procedures is shown in Fig. 1. In order to minimise burden upon the animals and minimise animal losses due to complications, an intramedullary nail model was used without any fracture or joint involvement. The study progressed from initial pilot studies, to establish surgical techniques, baseline antibiotic treatment and bacterial inoculation doses, to subsequent treatment groups that varied in terms of infecting pathogen (MSSA and MRSA) and application of local and/or systemic antibiotics.

In brief, the initial infection was created during the primary surgery by inoculating the IM canal of the tibia with either clinical MSSA or MRSA isolates, cultured from human patients. The inoculum was delivered in the tibia on a piece of collagen sponge placed through a mid-diaphyseal inoculation drill hole (see below for full details). After establishing a chronic ODRI, the two-stage revision of infected hardware began with a first revision surgery (RSx1) to remove the hardware, debride the IM canal and place a cement nail. Systemic antibiotic therapy commenced at this time point, in accordance with group assignment (Table 1). The second revision surgery (RSx2) was performed 2 weeks after RSx1 to remove the cement nail and place the definitive intramedullary (IM) nail. Systemic antibiotic therapy ceased at that time. A 4 weeks antibiotic washout period was observed after RSx2 in order to reduce risk of false negative culture results at euthanasia. Treatment groups are listed in Table 1.

Prior to commencement of the main study, two pilot studies were performed as shown in Table 1 . Three pilot animals were inoculated with MSSA and observed for 6 weeks, to allow a mature infection to establish. The first treatment group involved debridement and implant exchange without any antibiotic administration, in order to determine if 
debridement and implant exchange was sufficient to allow the sheep's immune defences to eradicate the remaining infection. The second pilot group received a complete treatment protocol including jet lavage at first revision surgery and gentamicin and vancomycin in the bone cement during the treatment period. Since all these sheep receiving a complete treatment protocol successfully eradicated the infection, it was decided that, in order to achieve a model of failed treatment (primary study goal), the animals in the main study would have a longer 8 weeks infection development period, would receive minimal debridement (no jet lavage) and only one antibiotic in the bone cement for MSSA-inoculated animals.

\section{Bacterial strains}

The MSSA strain used in this study was ATCC 25923 (hereafter called MSSA) and it was purchased from the German microorganism culture collection [Deutsche Sammlung von Mikroorganismen (DSM)] as strain DSM number No.1104. This MSSA is a human clinical isolate previously used in animal models of osteomyelitis (Schaer et al., 2012) and is susceptible to gentamicin, vancomycin and amoxicillin clavulanic acid. The MRSA strain used in this study was MRSA EDCC-5443 (DSM No.29134) (hereafter called MRSA). This MRSA is also a human clinical isolate and is resistant to amoxicillin calvulanic acid but susceptible to gentamicin, vancomycin and trimethoprim/ sulphadoxine.

\section{Bacterial inoculum preparation}

Cultures were taken from a frozen glycerol stock, streaked on tryptic soy agar plates (TSA, Oxoid AG, Pratteln, Switzerland) and incubated at $37^{\circ} \mathrm{C}$ for 16-18 h. A single colony of bacteria was picked from the agar, suspended in $25 \mathrm{~mL}$ of tryptic soy broth (TSB) and incubated for $14-17 \mathrm{~h}$ at $37^{\circ} \mathrm{C}$ under a $100 \mathrm{rpm}$ agitation. Then, the bacterial suspension was washed twice by centrifugation at $2500 \times g$ for $10 \mathrm{~min}$ and each time re-suspended in $20 \mathrm{~mL}$ sterile phosphate buffered saline (PBS, Sigma Aldrich, Buchs, Switzerland). Next, the bacterial suspension was exposed to ultrasonication for $3 \mathrm{~min}$ in an ultrasound water bath (Bandelin Sonorex Super $10 \mathrm{P}$, Bandelin, Berlin, Germany) operating at a frequency of $40 \mathrm{kHz}$ and once more vortexed for $30 \mathrm{~s}$. The optical density of the bacterial solution at $600 \mathrm{~nm}$ was adjusted to an OD ranging between 1.1-1.2, measured by spectrophotometry using a Multiskan ${ }^{\mathrm{TM}} \mathrm{GO}$ spectrophotometer (Thermo Scientific, Zürich, Switzerland). $20 \mu \mathrm{L}$ of this stock bacterial suspension were added to $1 / 8$ portion of a $3.6 \times 1.8 \mathrm{~cm}$ piece of collagen sponge (TissuFleece E, Baxter AG, Volketswil, Germany) to result in a total of approximately $2 \times 10^{7}$ colony forming units (CFU) per piece of collagen. Each animal received one freshly prepared piece of collagen inserted into the inoculation drill hole as described below. Quantitative culture of samples from each bacterial suspension was performed immediately after preparation by 10 -fold serial dilution and plating on

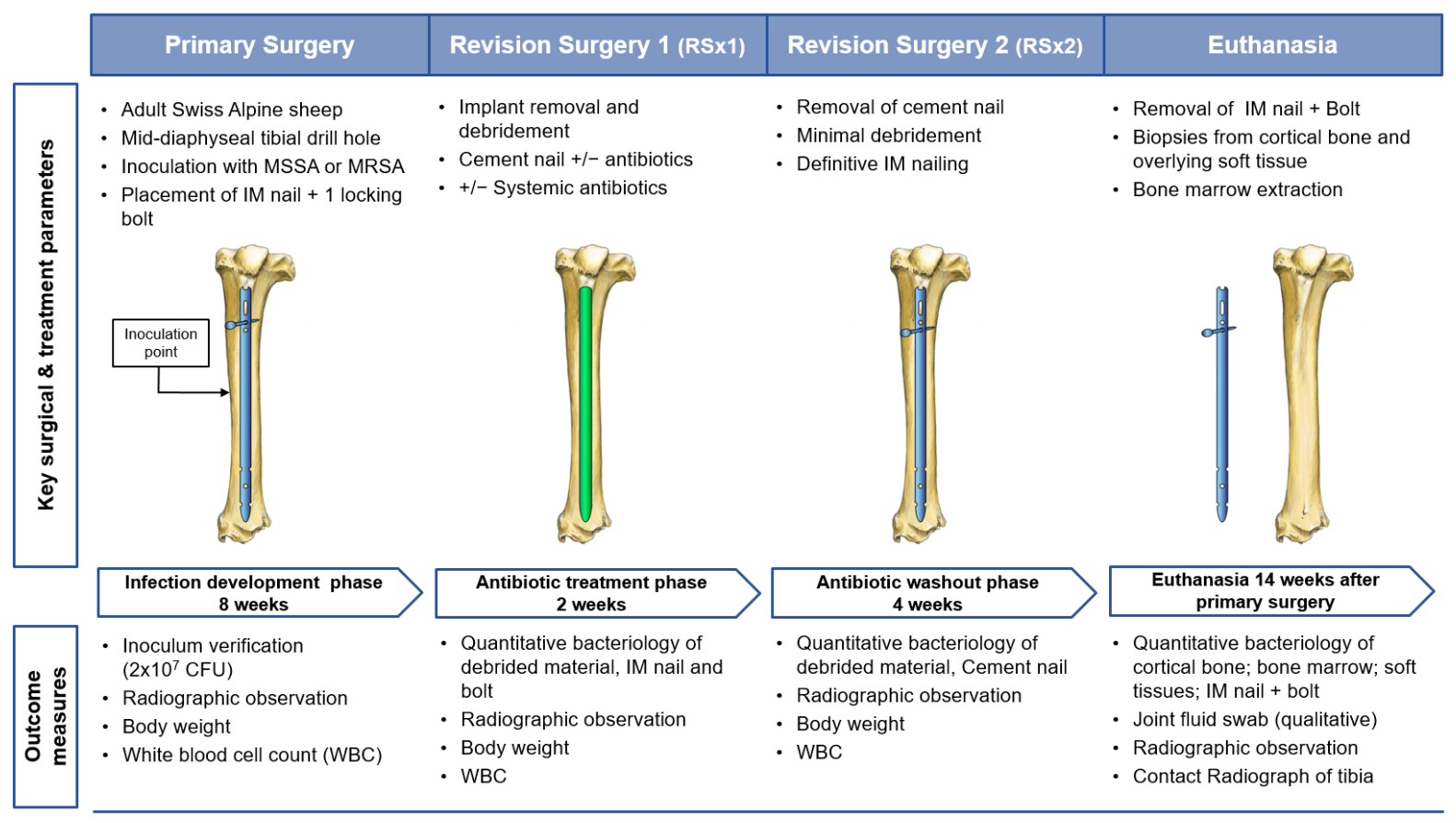

Fig. 1. Schematic overview of the infection development phase and subsequent treatment steps in the staged re-nailing of the infected tibial IM nail in the sheep. Upper text describes key surgical parameters at each surgical intervention and biopsy sampling at euthanasia. The lower text describes key outcome measures, including routine observations and quantitative bacteriological measures at each surgical intervention and at euthanasia. See Table 1 and text for complete details of group structure and local and systemic antibiotic therapy. 
Table 1. Overview of group structure including antibiotic treatment for MSSA (ATCC 25923) and MRSA (EDCC-5443)-infected animals. ${ }^{1}$ See text for full details of local and systemic antibiotics. ${ }^{*} 1$ sheep per group euthanised prior to RSx2 and data excluded from analysis.

\begin{tabular}{|c|c|c|c|}
\hline \multirow[t]{2}{*}{ Treatment / group } & \multicolumn{2}{|c|}{ Antibiotic therapy ${ }^{1}$} & \multirow[t]{2}{*}{$\mathbf{N}$} \\
\hline & Systemic & Local & \\
\hline \multicolumn{4}{|l|}{ Pilot } \\
\hline $\begin{array}{c}\text { No antibiotic (debride and } \\
\text { exchange alone) }\end{array}$ & None & None & 3 \\
\hline Systemic + local antibiotics & Amoxicillin clavulanic acid & Gentamicin & 3 \\
\hline \multicolumn{4}{|l|}{ MSSA } \\
\hline Systemic antibiotics & Amoxicillin clavulanic acid & None & 6 \\
\hline Local antibiotics & None & Gentamicin & $6^{*}$ \\
\hline Systemic + local antibiotics & Amoxicillin clavulanic acid & Gentamicin & 6 \\
\hline \multicolumn{4}{|l|}{ MRSA } \\
\hline $\begin{array}{l}\text { Systemic (as per MSSA group) } \\
+ \text { local antibiotics }\end{array}$ & Amoxicillin clavulanic acid & Gentamicin and vancomycin & $3^{*}$ \\
\hline $\begin{array}{l}\text { Systemic (pathogen adapted) } \\
+ \text { local antibiotics }\end{array}$ & Trimethoprim sulphadoxine & Gentamicin and vancomycin & 6 \\
\hline
\end{tabular}

TSA plates to check the actual total viable count of the prepared suspension.

\section{Antibiotic administration}

All sheep received ceftiofur [ $2.2 \mathrm{mg} / \mathrm{kg}$, intravenous injection (i.v.)] prior to the primary surgery. Ceftiofur is a third-generation cephalosporin licensed for use in veterinary medicine and was administered at the veterinary guideline dose for sheep. The antibiotic was administered after induction immediately prior to entry into the operating room. Additional doses were given during surgeries lasting longer than 90 min. Animals in the no antibiotic group also received preoperative ceftiofur prior to RSx1 and RSx2. Systemic antibiotic therapy involved either amoxicillin clavulanic acid or trimethoprim sulphadoxine, according to group assignment (Table 1). In all cases, systemic antibiotic therapy was given for $14 \mathrm{~d}$ between RSx1 and RSx2, with the initial dose given after the debridement in RSx1 and the last dose administered after completion of debridement in RSx2.

Amoxicillin clavulanic acid was administered subcutaneously once per day as Synulox ${ }^{\circledR}$ suspension (7.0 mg amoxicillin, $1.5 \mathrm{mg}$ clavulanic acid/kg; Zoetis Schweiz GmbH, Zurich, Switzerland), according to veterinary guidelines for sheep. Trimethoprim sulphadoxine was administered subcutaneously (s.c.) once per day as $24 \%$ Borgal $^{\circledR}$ suspension [12 mg sulphadoxine $+2.4 \mathrm{mg}$ trimethoprim $(3 \mathrm{~mL} / 50 \mathrm{~kg}$ body weight); MSD Animal Health $\mathrm{GmbH}$, Luzern, Switzerland]. Gentamicin-loaded cement nails were prepared with Palacos ${ }^{\circledR} \mathrm{R}+\mathrm{G}$ (0.5 g per $40 \mathrm{~g}$ of cement); cement nails with gentamicin and vancomycin were prepared with $\mathrm{COPAL}{ }^{\circledR} \mathrm{G}+\mathrm{V}(0.5 \mathrm{~g}$ gentamicin and $2 \mathrm{~g}$ vancomycin per $40 \mathrm{~g}$ of cement); non-antibioticloaded bone cement nails were prepared with PALACOS $^{\circledR} \mathrm{R}$ (all cements were purchased from Heraeus, Hanau, Germany). The antibiotic loading amount was performed according to manufacturer's instructions.

\section{Surgical anaesthesia and analgesia protocol (all surgeries)}

Animals were sedated with $0.04 \mathrm{mg} / \mathrm{kg}$ of detomidine (Domosedan ${ }^{\circledR}$, Provet AG, Lyssach, Switzerland) approximately $20 \mathrm{~min}$ before transfer to the preparation area. Each sheep received $0.2 \mathrm{mg} /$ kg (i.v.) of diazepam (Valium ${ }^{\circledR}$, Roche Pharma AG, Switzerland) and $4 \mathrm{mg} / \mathrm{kg}$ (i.v.) of ketamine (Ketasol-100 ${ }^{\circledR}$, Roche Pharma AG) for induction. After endotracheal intubation, anaesthesia was maintained using isoflurane (Isofluran Baxter ${ }^{\circledR}$, Baxter AG) in oxygen and air. Then, the sheep received an epidural injection with $2 \mathrm{mg} / \mathrm{kg}$ of lidocaine (Lidocain ${ }^{\circledR} 2 \%$ Streuli, Streuli Pharma AG, Uznach, Switzerland) mixed with $0.005 \mathrm{mg} / \mathrm{kg}$ of burprenorphine (Temgesic $^{\circledR}$, Reckitt Benckiser AG, Switzerland). For subsequent systemic analgesia, the sheep received a non-steroidal anti-inflammatory drug [4 mg/kg (i.v.) of carprofen (Rimadyl ${ }^{\circledR}$ Rind, Zoetis Schweiz $\mathrm{GmbH})$ ] and opioids [intramuscular injection (i.m.) of $0.05 \mathrm{mg} / \mathrm{kg}$ of buprenorphine (Temgesic ${ }^{\circledR}$, Reckitt Benckiser AG)]. The postoperative analgesia protocol was $4 \mathrm{mg} / \mathrm{kg}$ subcutaneous injection (s.c.) of carprofen (Rimadyl ${ }^{\circledR}$, Pfizer AG, Zürich, Switzerland), every $48 \mathrm{~h}$ for a minimum of $3 \mathrm{~d}$, and $0.05 \mathrm{mg} / \mathrm{kg}$ i.m. of buprenorphine (Temgesic ${ }^{\circledR}$, Reckitt Benckiser AG) every $8 \mathrm{~h}$ for $24 \mathrm{~h}$, followed by $2 \mu \mathrm{g} / \mathrm{kg} / \mathrm{h}$ of fentanyl (Durogesic $^{\circledR}$ Matrix patches, Janssen-Cilag AG, Zug, Switzerland) for $72 \mathrm{~h}$. Carprofen was continued every other day as deemed necessary by the attending veterinarian.

\section{Primary surgery}

After induction of anaesthesia, animals were placed in dorsal recumbency. The left tibia and knee joint were prepared and draped for aseptic surgery. A 
$1 \mathrm{~cm}$ incision was made on the medial side of the proximal tibial diaphysis, $12 \mathrm{~cm}$ distally to the tibial plateau, and a $4.3 \mathrm{~mm}$ unicortical hole $(4.3 \mathrm{~mm}$ drill bit, Depuy Synthes, Bridgewater, NJ, USA) was drilled through the medial cortex, to allow the introduction of the bacterial inoculum on the collagen sponge (inoculation drill hole). Then, the tibial plateau was approached by a longitudinal incision along the patellar ligament (medial para-patellar approach). The incision started proximally, distal to the patella along the patellar ligament, down to the tibial tuberosity (approximately $5 \mathrm{~cm}$ ). Next, the stifle joint was flexed to approximately $90^{\circ}$, in order to gain access to the tibial plateau. The entry point for the nail was cranial to the stifle joint, on a line between the patellar tendon and the anterior edge of the medial meniscus (Fig. 2A). A K-wire (1 mm, Depuy Synthes) was inserted through the planned entry point along the anatomical axis of the tibia under fluoroscopic control to check for positioning and alignment (Fig. 2B). A cannulated awl (10 mm, Depuy Synthes) was placed over the guiding K-wire and the medullary canal was opened using a twisting motion. After removal of guide wire and awl, a $190 \mathrm{~mm}$ long $7.5 \mathrm{~mm}$ diameter unreamed solid humeral nail (UHN, Depuy Synthes) was inserted by pushing and gentle rotation on the insertion handle. The UHN nail was selected amongst the range of commercially available nails, as it was deemed to provide the best fit for the sheep tibia. The nail was placed as distally as possible in the bone marrow cavity. Correct insertion was controlled with fluoroscopy. Using the insertion handle as an aiming device, the more distal of the two proximal $3.9 \mathrm{~mm}$ locking bolt holes received an interlocking bolt (3.9 mm, Depuy Synthes) by a stab incision through the skin after predrilling $(3.2 \mathrm{~mm}$ drill bit, DePuy Synthes, Oberdorf, Switzerland). The fascia and subcutaneous tissues above the nail entry point and bolt-hole incision were closed with absorbable suture material in a continuous adapting pattern. The skin at these locations was closed with an intradermal suture (\#3-0 MONOCRYL, Ethicon, Sommerville, NJ, USA). The bacterial inoculum was placed in the inoculation drill hole on a piece of collagen sponge (20× TissuFleece E, Baxter AG), so that it directly contacted the implant and filled the inoculation drill hole. The collagen was used to control the location of the bacteria in the early hours post-
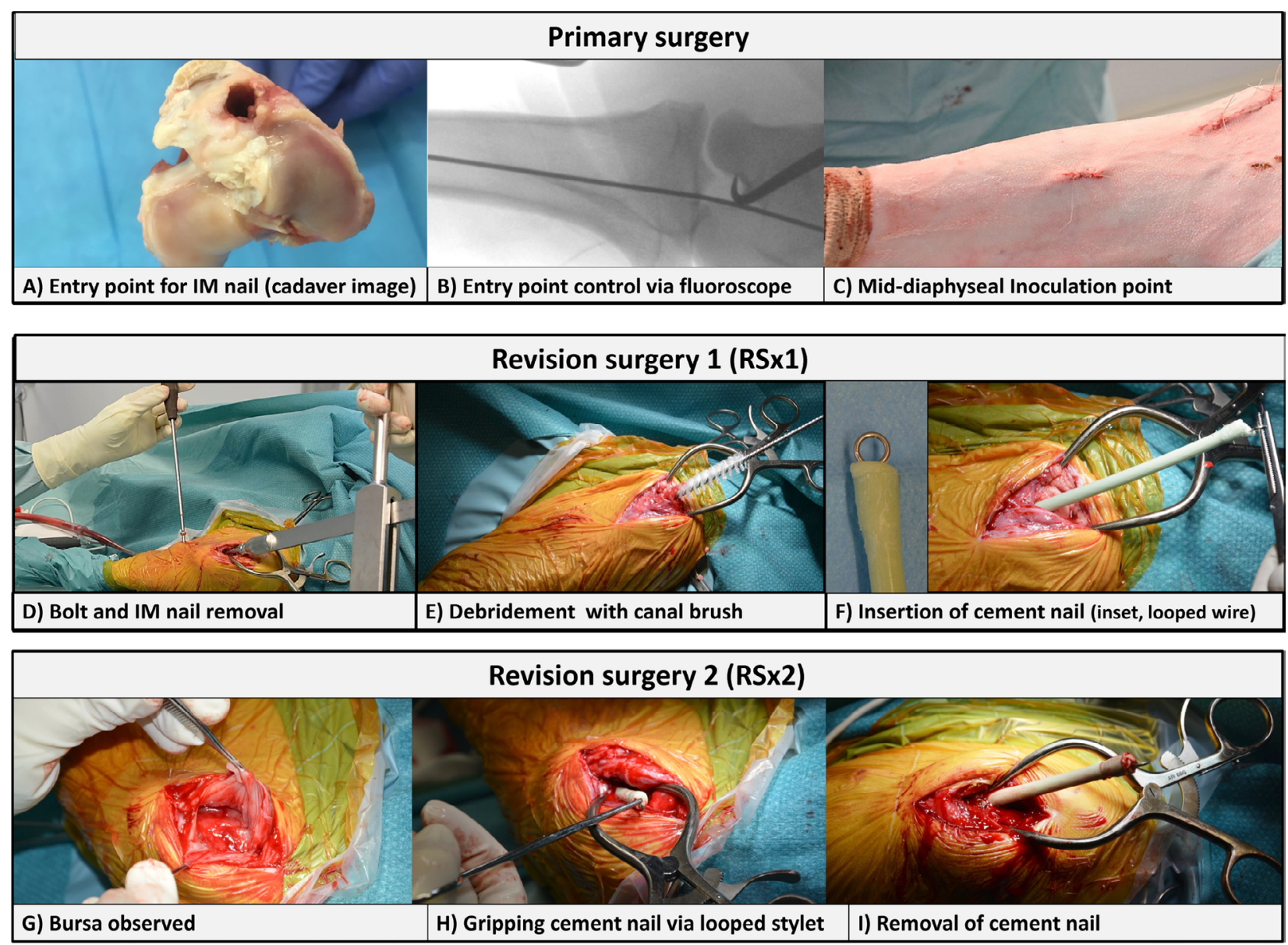

Fig. 2. Intraoperative images showing critical features of the primary surgery (upper panel), revision surgery 1 (middle panel) and revision surgery 2 (lower panel). Primary surgery involved identification of nail entry point, correct placement of hardware under fluoroscopic control and inoculation of bacteria into the mid-diaphyseal bone through a separate incision. Revision surgery 1 involved removal of IM nail and bolt with subsequent debridement and placement of a cement nail. Revision surgery 2 involved removal of the cement nail, definitive fixation with a new sterile IM nail and placement of new bolt in a new bolthole. 
surgery and maximise the likelihood of a localised bone infection. The collagen is expected to degrade within the 8 weeks initial observation period and, as such, would not influence the treatment protocols and merely served to aid in the establishment of the infection. Subsequently, the soft tissues overlying the inoculation drill hole were closed with an intradermal suture as described above, but the drill hole in the bone was not itself sealed (Fig. 2C). The proximal incisions were covered with a stent for $3 \mathrm{~d}$. The limb was bandaged for $3 \mathrm{~d}$ or longer with twice-weekly bandage changes in case of wound healing problems.

\section{Revision surgery 1 (RSx1)}

After induction of anaesthesia, animals were again placed in dorsal recumbency. The left tibia and knee joint were prepared and draped for aseptic surgery. The entry point in the tibial plateau was approached the same way as described in the initial surgery. The entry point was reopened, with a cannulated awl, to a diameter of $10 \mathrm{~mm}$, a swab was taken for bacterial culture and the nail extraction device was screwed into the head of the nail. The locking bolt was removed through a stab incision, followed by removal of the nail (Fig. 2D). Debridement of the IM cavity of the tibia was performed by using a femoral canal brush (Smith and Nephew AG, London, UK) inserted through the entry point, pushed as far distally as possible, turned $90^{\circ}$ and removed (Fig. 2E). The brush was sampled for quantitative bacteriology as described in the quantitative bacteriology section below. Removed hardware and debrided materials were also taken for quantitative bacteriology.

A cement nail was fabricated by encasing a $1.1 \mathrm{~mm}$ stainless steel cleaning stylet (DePuy Synthes, Switzerland) in polymethylmethacrylate (PMMA, Heraeus). Antibiotics incorporated and manufacturer details are described in the antibiotic administration section. The preformed loop was used at the proximal end of the spacer and an additional loop was created at the distal end to facilitate simple and complete removal of the cement nail. The stylet was also bent to approximate the bend of the humeral nail and then placed in a $6.5 \mathrm{~mm}$ endotracheal tube, as shown in Fig. 2F (Rüsch, Belp, Switzerland). Then, the cement was prepared according to manufacturer's instructions and added to the tube. The length of the cement nail was customised according to the length of the tibial canal of each sheep, as measured radiographically, in order to ensure the entire tibial cavity was adjacent to the cement nail.

After the cement had fully hardened, the cement nail was removed from the tube and stored in a sterile container until insertion in the medullary cavity (Fig. 2F). A gentamicin collagen sponge (Garamycin ${ }^{\circledR}$ Schwamm, Curatis AG, Liestal, Switzerland) was placed in the parapatellar incision of all animals before closure, to minimise the risk of superficial incisional infections. The local release of gentamicin in the superficial tissue was not expected to reach bactericidal concentrations inside the IM canal or influence the progression of infection within the bone. Closure, bandaging and pain management were performed as described for the initial surgery.

\section{Revision surgery 2 (RSx2)}

Preparation and approach to the entry point were the same as described for the initial surgery. The cement nail was removed and sampled for microbiological analysis, as described below (Fig. 2H,I). Samples of the bone marrow were taken with a curette, followed by insertion of a new sterile solid $190 \mathrm{~mm} 7.5 \mathrm{~mm}$ UHN DePuy Synthes nail. The more proximal of the two proximal locking bolt holes (not used in primary surgery) received an interlocking bolt (3.9 mm Depuy Synthes) after a stab incision and drilling of a new $3.2 \mathrm{~mm}$ hole (DePuy Synthes, Switzerland), using the insertion handle as aiming device. Closure, bandaging and pain management were done as described for the initial surgery. Removed hardware and debrided materials were taken for quantitative bacteriology.

\section{Animal welfare and observation}

Stents were removed after $3 \mathrm{~d}$ and bandages removed after 3-5 d or changed twice weekly for as long as necessary. Incision sites were checked at each bandage change. The sheep were kept in groups of 2-3. The animals were routinely checked by a veterinarian or an experienced animal caretaker for general behaviour, weight bearing on the operated limb, body temperature, respiration, appetite and defecation. Animal weights were recorded before surgery and then every other week. Euthanasia was done with an intravenous overdose of pentobarbital (Esconarkon ${ }^{\circledR}$, Streuli Pharma AG). Any animals displaying systemic signs of infection or ongoing lameness, elevated temperature, WBC or loss of more than $10 \%$ body weight in one month were considered for early euthanasia. A customised cumulative clinical scoring system was used to objectively asses the burden based upon these key parameters. Reaching predefined scores resulted in euthanasia of the animal.

\section{Radiography}

Mediolateral and craniocaudal radiographic projections of the left tibia of each sheep were taken before each surgery, immediately after each surgery, then every other week and at euthanasia. Sheep were sedated for radiographs $(0.04 \mathrm{mg} / \mathrm{kg}$ i.m. of detomidine; E. Graeub AG, Bern, Switzerland). Additionally, a high-resolution contact radiograph (full thickness) was taken post-mortem, using highresolution technical film (D4 Structurix DW ETE, $\mathrm{Agfa}^{\circledR}$, Belgium) and a cabinet $\mathrm{X}$-ray system (Model No. 43855A, Faxitron X-Ray Corporation ${ }^{\circledR}$, USA) at $42 \mathrm{kV}$ for $5 \mathrm{~min}$, with a $0.5 \mathrm{~mm}$ aluminium filter.

\section{Haematology}

Blood samples were taken preoperatively, $3 \mathrm{~d}$ postoperatively and once weekly thereafter. 
Whole blood was collected from the jugular vein using disposable needles and EDTA containing vacutainer tubes (S-Monovette, Sarstedt AG, Sevelen, Switzerland). Total white blood cell count (WBC) was performed on whole blood samples within $1 \mathrm{~h}$ after collection using an electronic cell counter (Vet ABC, Scil animal care, Viernheim, Germany). Erythrocyte sedimentation rate (ESR) and C-reactive protein (CRP) were measured in infected and non-infected animals in the pilot study. Measurements were discontinued due to lack of any correlation between values and infection status.

\section{Post-mortem macroscopic evaluation}

A post-mortem macroscopic examination of the external body surface and surgery sites was conducted on all animals. After euthanasia was complete, the initial dissection of the operated limb, the tibia with adjacent limbs and overlying musculature and skin, were frozen at $-20^{\circ} \mathrm{C}$, in order to facilitate the bacteriological processing within one working day. This protocol is known to retain viability of bacteria based on ex vivo simulation testing. On the day of bacteriological processing, the skin and fur were sprayed with $70 \%$ ethanol, carefully dissected away and the exposed underlying tissue was once again wiped down with $70 \%$ ethanol. Swabs were immediately taken from joint, entry point of the IM nail into the tibia, interlocking bolt and drill-hole site. In all cases, swabs were taken with care to prevent contamination from skin and wool. The swabs were streaked onto blood agar (BA) plates prepared using Blood Agar Base (Oxoid AG, Pratteln, Switzerland), containing $5 \%$ defibrinated horse blood (TCS Biosciences Ltd., Buckingham, UK).

\section{Quantitative bacteriology of debridement} materials, hardware, and post-mortem biopsies All samples for bacteriological examination were taken with careful attention to aseptic technique. The interlocking bolt and the IM nail at RSx1 and at euthanasia, as well as the cement nail at RSx2, were carefully removed intraoperatively. The interlocking bolt was placed into a $50 \mathrm{~mL}$ Falcon tube containing $20 \mathrm{~mL}$ sterile PBS and vortexed for $30 \mathrm{~s}$. The IM nail, the femoral canal brush and the cement nail were placed in sterile glass tubes containing $60 \mathrm{~mL}$ sterile PBS, sealed and agitated/inverted 10 times. All hardware samples were subsequently placed in the sonicating water bath operating at a frequency of $40 \mathrm{kHz}$ for $5 \mathrm{~min}$ and once more vortexed for $30 \mathrm{~s}$ or agitated 10 times.

Bone marrow samples that were collected at RSx2 and euthanasia were weighed and homogenised in $20 \mathrm{~mL}$ PBS using an Omni-TH hand-held homogeniser (LabForce AG, Switzerland) with sterile Omni-tip plastic probes. Cortical bone biopsies were taken at euthanasia from the empty interlocking bolt holes (distal and proximal) and the inoculation point using a Partsch spoon, weighed and homogenised in $20 \mathrm{~mL}$
PBS using the Omni-TH hand-held homogeniser with sterile Omni-tip plastic probes.

All hardware sonicates and tissue homogenates were 10-fold serially diluted in sterile PBS and $200 \mu \mathrm{L}$ were spread onto BA plates. Agar plates were incubated at $37^{\circ} \mathrm{C}$ and colonies counted at 24 and $48 \mathrm{~h}$. The lower limit of detection (LLOD) for bacteria was $100 \mathrm{CFU} /$ bone fragment, $100 \mathrm{CFU} /$ bone marrow, $100 \mathrm{CFU/bolt} \mathrm{and} 300 \mathrm{CFU} / \mathrm{IM}$ nail/cement nail/femoral brush. Viable CFU counts were obtained from each individual sample; however, to simplify data presentation, data from different biopsies of the same material/source were consolidated into single values. Specifically, this entails: CFU counts of IM nail and interlocking bolt were combined as a single IM nail + bolt count; proximal and distal bone marrow were combined into a single bone marrow count; cortical bone samples from bolt-holes and inoculation point were combined into a single cortical bone count. To be considered infected, an animal was required to yield at least ten times the LLOD in at least one biopsy.

Bacterial growth from each animal was evaluated by a $S$. aureus-specific latex agglutination test (Staphaurex Plus, Remel, Basel, Switzerland). A single representative colony from each infected animal was typed by Random Amplified Polymorphic DNA (RAPD), according to the method of Morandi et al. (2009), to confirm it was the inoculated S. aureus strain and exclude cross contamination or infection with another $S$. aureus strain.

\section{Statistical evaluation}

Primary outcome measure was infection rate in each study group and two-sample comparisons were made using the two-tailed Mann-Whitney rank sum test. The group size was set at 6 in order to determine statistically significant differences greater than $70 \%$ between treatment groups. Animals in the pilot groups and the MRSA group not receiving the pathogen-adapted treatment were excluded from statistical analysis due to smaller group sizes. Weight and WBC measurements were compared by two-way ANOVA with repeated measures. For all tests, $p$ values $<0.05$ were considered statistically significant.

\section{Results}

\section{Animal welfare}

Overall, the sheep recovered well after each surgery. Lameness was transient or, if persistent, led to early removal from the study (complications are listed below). Between RSx1 and RSx2, the sheep tended to develop a bursa in the incisional area between the patellar tendon and the subcutaneous tissue (Fig. $2 \mathrm{G})$. The bursa did not cause any problems, such as lameness or compromised wound healing. None of the sheep developed complications at the parapatellar incision. The removal of the nail or cement nail was 


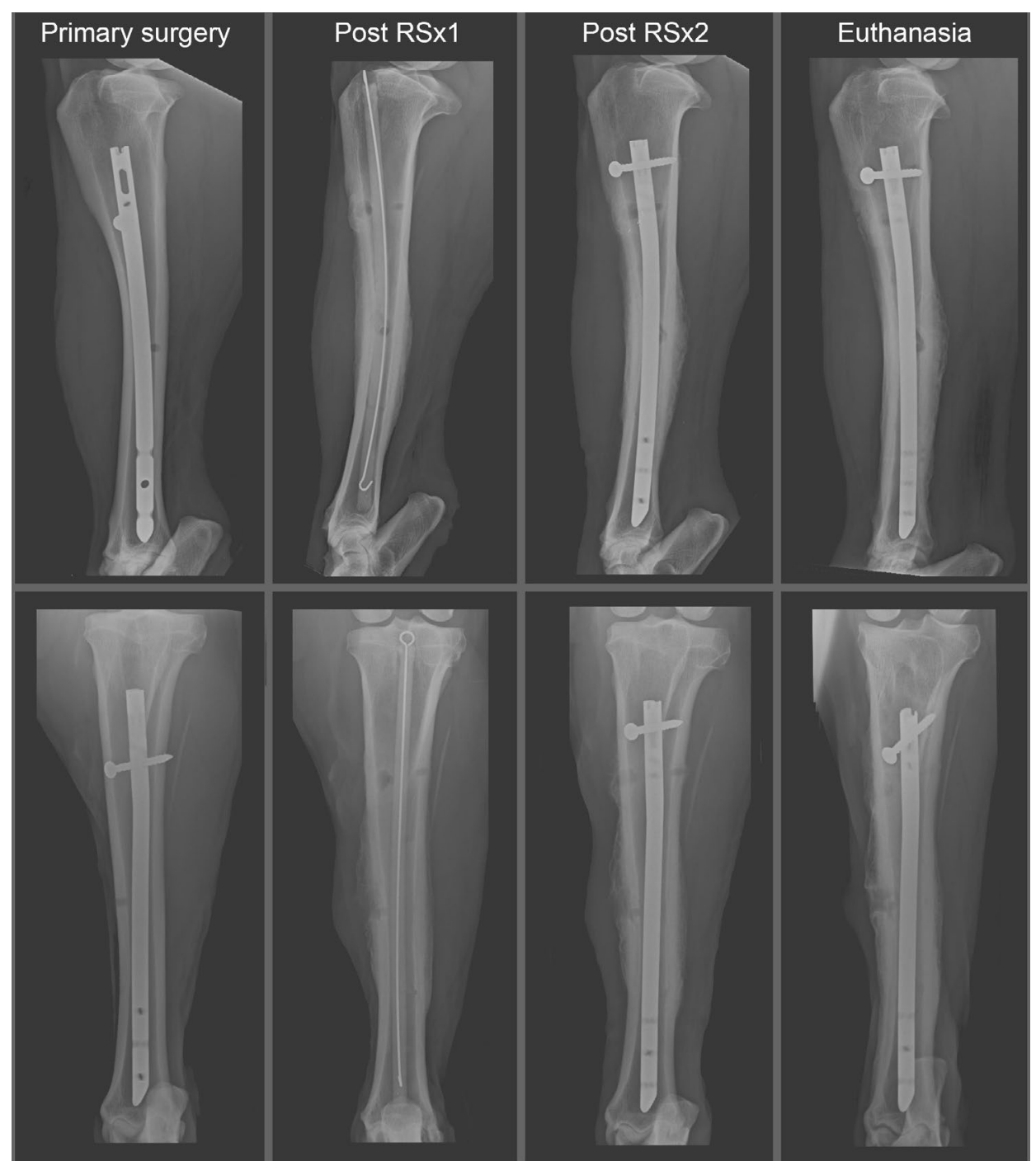

Fig. 3. Representative radiographs illustrating the progression of one sheep through each stage of the model from primary surgery, through RSx1, RSx2 and euthanasia. The images were taken from a sheep in the MRSA group receiving a gentamicin and vancomycin-loaded cement nail and systemic trimethoprim and sulphadoxine (treatment failed, see Fig. 5). Images show the mediolateral projection (upper row) and craniocaudal projection (lower row) at each stage of the study protocol. Note: the use of the second-most proximal bolt-hole in RSx1 and the most proximal bolt hole in RSx2.

performed in all sheep without difficulties. Postoperative radiographs from each surgery and at euthanasia for a representative sheep receiving MRSA inoculum and trimethoprim sulphadoxine treatment are shown in Fig. 3. Animals were euthanised at the scheduled euthanasia, which was 4 weeks after RSx2, unless otherwise stated.

\section{Complications and exclusions}

Two sheep were euthanised during the initial infection development phase for problems unrelated to the infection: one due to oesophageal obstruction and a second due to a closed complete spiral fracture over the length of the diaphysis of the tibia. All further complications either did not require early euthanasia or occurred after RSx2.

During the initial infection development phase after primary surgery, 3 sheep developed a fistula overlying the point of bacterial inoculation, with purulent discharge. The wounds were cleaned externally and bandages changed regularly. The discharge was self-limiting in all cases and resolved prior to revision surgery without any additional treatment. The other deviation from the scheduled interventions occurred in 5 animals after RSx 2 due to continuing weight loss or lameness. This included all 3 sheep of the no antibiotic group and 2 of the 6 sheep of the local antibiotic group. These sheep experienced more than $10 \%$ weight loss, increasing lameness and clear signs of infection in the first 2 weeks after RSx2. According to our ethical approval conditions, these sheep were euthanised prior to scheduled euthanasia ( 2 weeks early). Since these animals had received the full surgical and medical treatment at this stage, they were not excluded from the study and results were included in the final analysis.

\section{Observation}

Change in body weight for all sheep at RSx1, RSx2 and at euthanasia is shown in Fig. 4. Overall, there was a general trend for weight loss; however, there were a few statistically significant changes. At RSx1 there were no statistically significant differences from baseline values. By RSx2, the sheep receiving systemic and local antibiotics displayed significant weight loss $(p<0.05$ relative to pre-operative weight), although these animals recovered much weight thereafter. At euthanasia, the local antibiotic group lost the most weight ( $p<0.05$ relative to pre-operative weight). White blood cell (WBC) counts were also recorded 
throughout the observation period and outlined in Fig. 4. There was substantial variation within the groups at each time-point, leading to a lack of any distinct pattern, either within the groups over time or between the groups or due to infection status at any time point. Both the local and systemic treatment groups in the MSSA infection arm displayed increased WBC at euthanasia (the no antibiotic group was excluded from statistical evaluation due to small group size and pre-term euthanasia).

Post mortem high-resolution contact radiography High-resolution contact radiographs of the operated tibia from all sheep were taken at euthanasia (Fig. 5). The radiographs of all groups exhibited signs of hyperostosis. Irregular bone structure with lytic areas could be seen in all cases, especially in the more distal part of the tibia. There was lysis around the inserted locking bolt and markedly reduced or no new bone in the hole of the distal locking bolt. Signs of sequestrum formation were seen in the image from the no antibiotic group. In contrast, the radiograph of the systemic and local antibiotics (MSSA) group showed comparatively moderate thickening of the mid-diaphyseal cortical bone, but no signs of active osteomyelitis and the most distal of the used drill holes, which was removed 6 weeks before euthanasia, was filled in with new bone.

\section{Bacteriological evaluation}

The bacterial inoculum given to each sheep was within our target range of 1-2 × $10^{7} \mathrm{CFU}$ per animal, as revealed by quantitative culture (data not shown). A summary of the quantitative bacteriology data from the biopsies and hardware sampled at each surgery and at euthanasia, is shown in Fig. 6. All sheep were found to be infected at RSx1, regardless of whether the animal was initially inoculated with MSSA or MRSA. Furthermore, at this stage, there was no significant difference between the infectious burden between any particular group or between MSSA and MRSA inoculated animals $(p>0.5)$. The bacteriological evaluation at RSx2 was performed on samples from sheep that were under antibiotic treatment. Therefore, any culture negative result at this time should consider that there might be some inhibitory effects masking true positive samples, although the samples were processed in large volumes of diluent, which should dissipate the effective concentration of any antibiotic and further

A

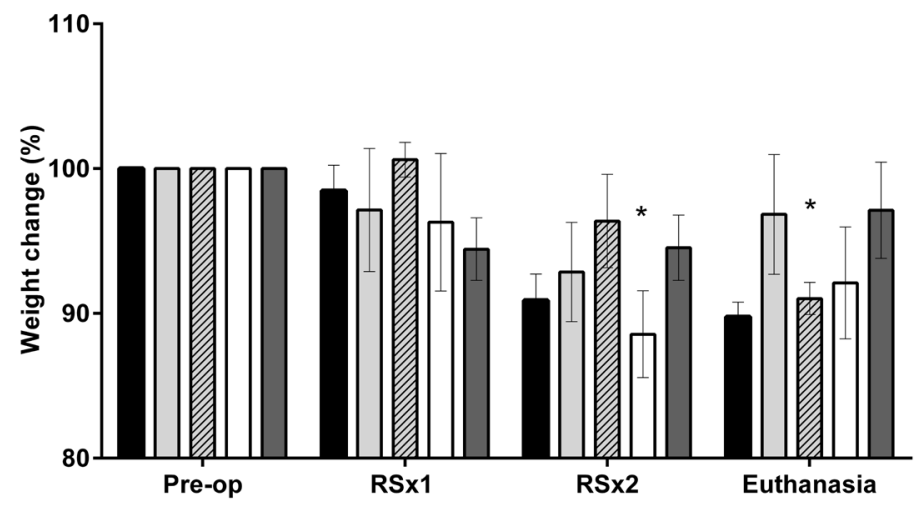

B

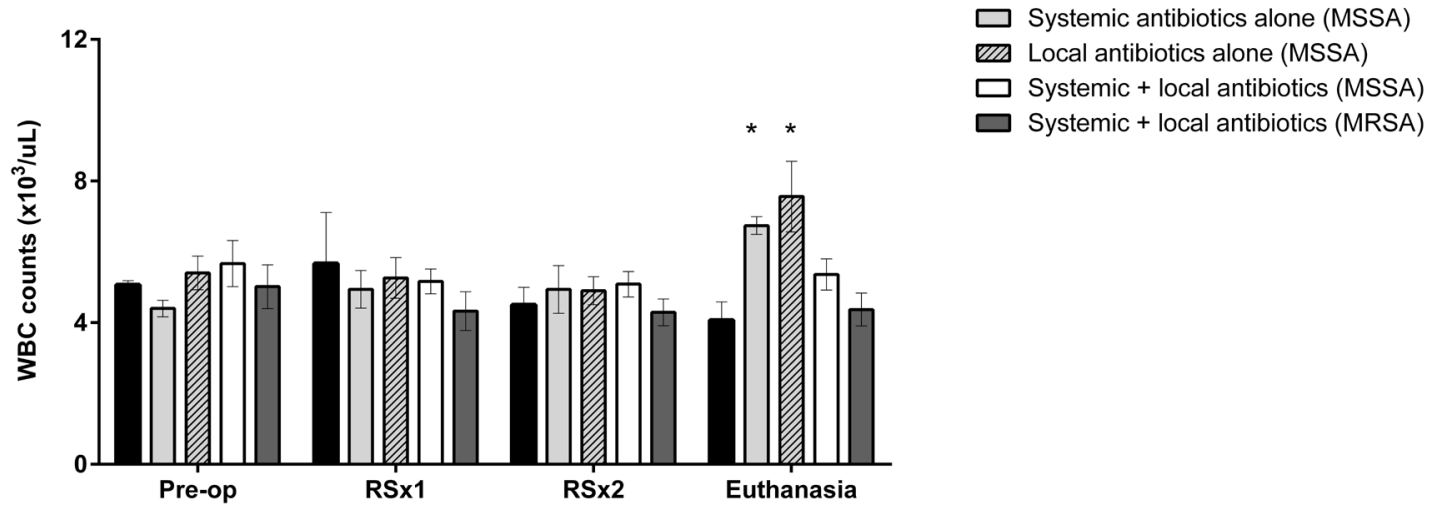

Fig. 4. Relative change in body weight (above) and white blood cell count (WBC, below) of each treatment group throughout the observation period. Data presented as mean \pm standard error of the mean $\left({ }^{*} p<0.05\right.$ relative to starting weight or starting WBC). Note that the no antibiotic group was excluded from statistical analysis due to small group size and due to the fact that these animals were euthanised prior to scheduled euthanasia time point. 
placement on agar plates would result in further dilution of the antibiotic.

All bacteria isolated from infected sheep were found to be $S$. aureus by latex agglutination test and selected strains were confirmed to be identical to the inoculated S. aureus strain by RAPD PCR (data not shown). In two cases, bacteria other than $S$. aureus (based on colony morphology) were identified in superficial biopsies at euthanasia. In all cases, the colony count of such isolates was low and displayed a variety of colony types indicative of contamination from overlying skin or wool and thus were not considered as invasive infections. In all cases, swabs were only found to be positive in animals yielding quantifiable CFU data. There were no incidences of a positive swab culture and negative quantitative culture. Swab results are therefore not shown and only the quantitative CFU data are presented below.
Treatment of MSSA infection

All 3 sheep in the no antibiotic group experienced lameness and weight loss that continued after RSx2, thus they were euthanised earlier than scheduled. At euthanasia (Fig. 6A), all samples were again culture-positive, confirming that debridement and device exchange alone could not treat the infection in this model.

In all 6 animals in the systemic antibiotic treatment group both debrided bone marrow and the IM nail were culture-negative at RSx2 (Fig. 6B). However, bacterial recovery and growth was observed at euthanasia for all 6 sheep, indicating antibiotic therapy was insufficient for eradication of all the bacteria, despite the culture negative status at RSx2.

Five animals in the local antibiotic only group completed the three surgeries. Bacterial growth was recovered at RSx2 from one cement nail and from
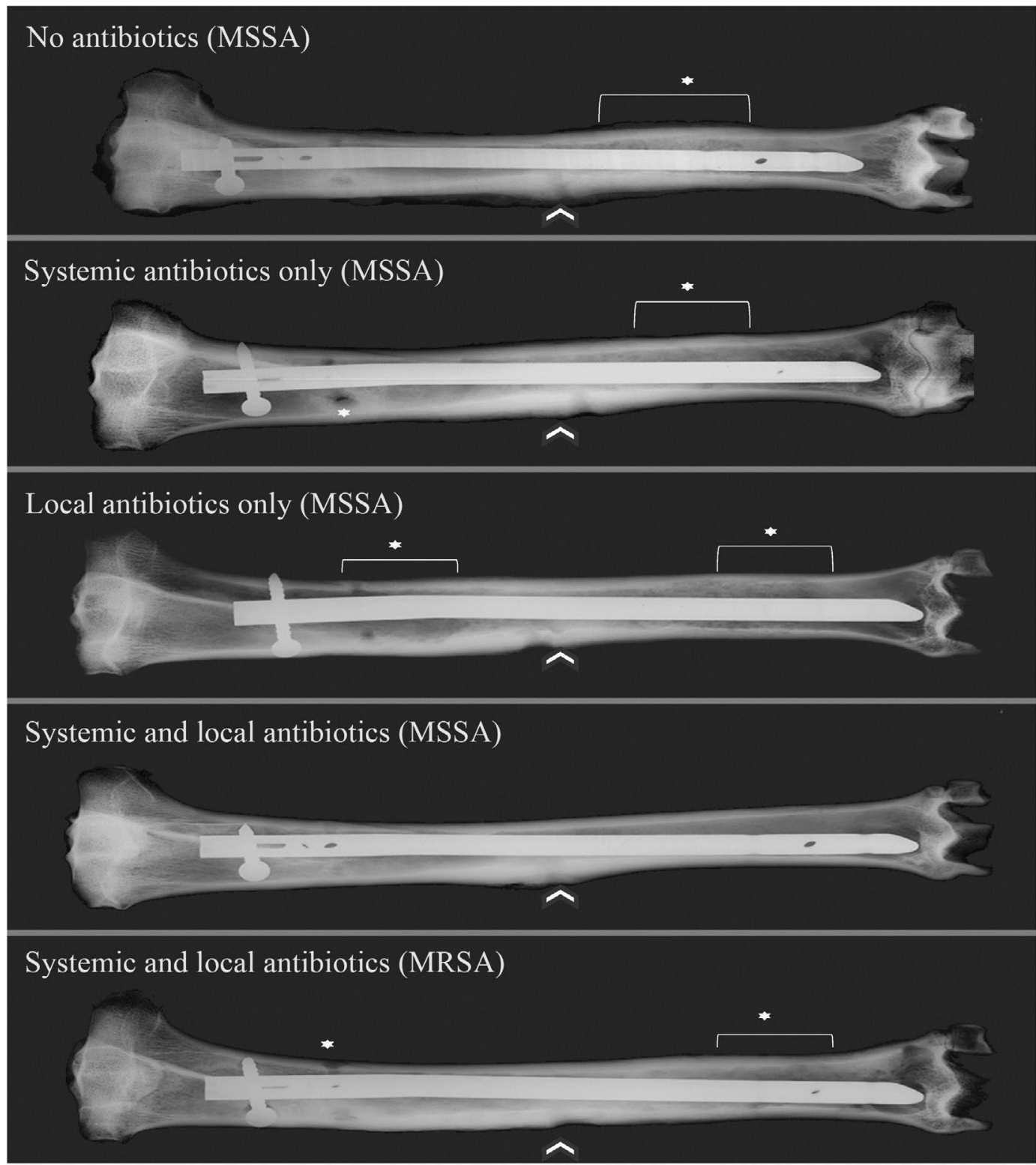

Fig. 5. Representative post-mortem contact radiographs of sheep of each treatment group at euthanasia. The radiograph in the MRSA group was taken from an animal receiving trimethoprim sulphadoxine. Signs of osteomyelitis were seen in all groups at the inoculation drill hole (white arrows) and at other locations (asterisk). Note that the systemic and local antibiotics (MSSA) group displayed a more physiological appearance. 
A) No antibiotics (MSSA)

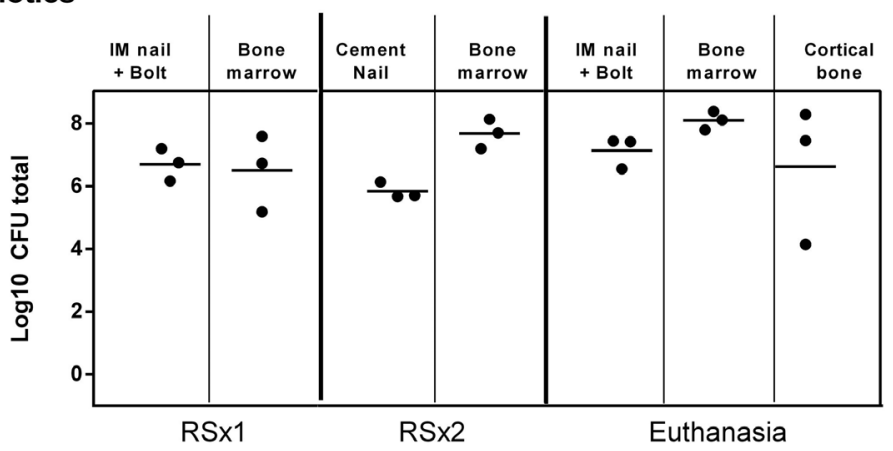

B) Systemic antibiotics alone (MSSA)

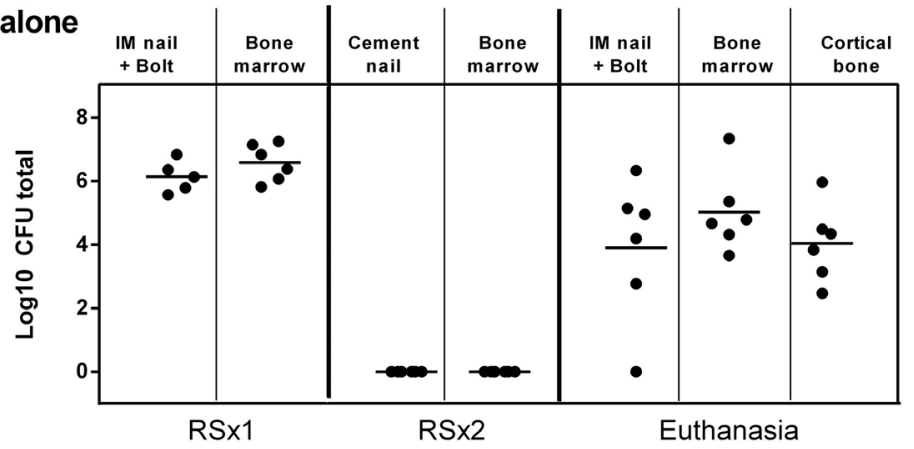

C) Local antibiotics alone (MSSA)

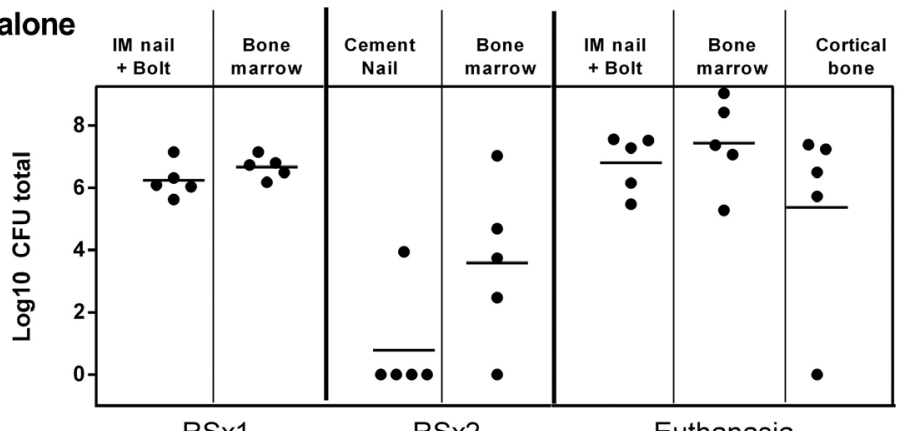

D) Systemic +

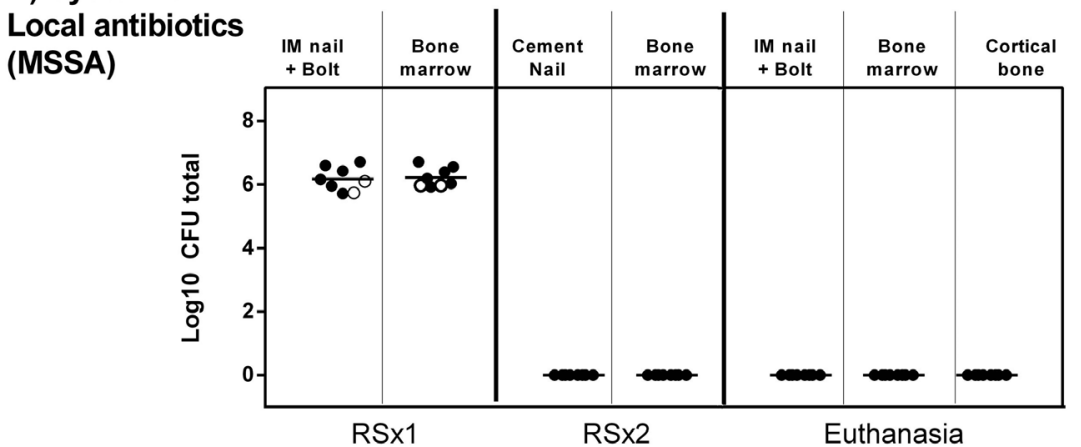

E) Systemic + Local antibiotics (MRSA)

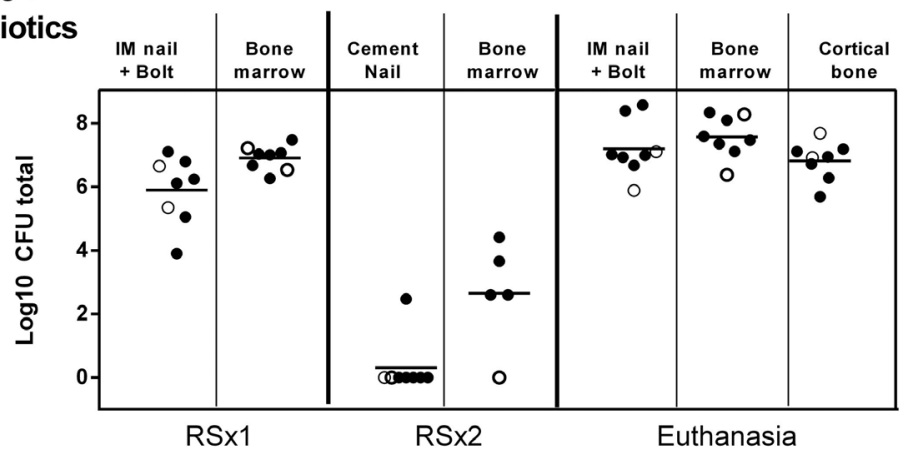

Fig. 6. Quantitative bacteriology from hardware and tissue samples at $\mathrm{RSx} 1, \mathrm{RS} \times 2$ and euthanasia. See materials and methods for description of data consolidation. In D, the three sheep with shorter infection phase and additional debridement are identifiable by empty circles. In E, the three sheep receiving amoxicillin clavulanic acid are identifiable by empty circles. Lines represent mean values. 
the bone marrow of 4 sheep (Fig. 6C). The infection then remained/recovered in all 5 sheep, with culture positive samples at euthanasia.

A total of 6 sheep inoculated with MSSA received systemic and local antibiotic treatment. As described in Table 1, we additionally operated on 3 pilot sheep who received a slightly shorter infection phase (6 weeks) and modified debridement and local antibiotics (white circles, Fig. 6D). One of these 3 sheep was euthanised prior to RSx2, as described above, and data were excluded from analysis. All the other animals in this group, regardless of treatment, were culture negative at RSx2 and at euthanasia.

\section{Treatment of MRSA infection}

The MRSA-inoculated animals received systemic and local antibiotic treatment. Treatment was performed in two groups (Table 1): amoxicillin clavulanic acid was selected for an initial smaller group, in order to facilitate direct comparison with MSSA treatment. A second, larger treatment group received trimethoprim and sulphadoxine, as a pathogenadapted treatment, due to the susceptibility of the utilised strain to this antibiotic. Data from animals receiving both treatments were displayed together within the same group (Fig. 6E; amoxicillin clavulanic acid-treated group white circles, trimethoprim and sulphadoxine group black circles). After treatment with local and systemic antibiotics, the infection remained/recovered in all 8 sheep, regardless of antibiotic selection.

\section{Discussion}

Currently, a staged exchange of infected hardware is the preferred option for many patients with ODRI. The relatively high rate of treatment failure, despite the radical nature of the two-stage exchange, indicates that current practices are sub-optimal. In order to improve treatment outcomes in the future, a better understanding of the reasons underlying this failure is required. In this study, we have established the first large animal model of a staged exchange of infected orthopaedic hardware, in order to provide a model for failed therapy, as a testbed for studying current or possible future therapeutic strategies. This was the first model that encompassed: 1) a clinically relevant 8 weeks chronic infection with bacterial colonisation of the device and radiographic signs of osteomyelitis at revision; 2) devices and basic debridement tools as used for human surgery; 3) local and/or systemic antibiotic therapy on a scale similar to human regimens; 4) quantitative bacteriological outcomes at each stage of the treatment.

Despite the fact that large animal models are more expensive and complex than smaller animal models, we nevertheless have pursued a large animal model due to the opportunity to utilise actual clinically available human implants and surgical instruments, which are not applicable in smaller animals. Furthermore, since sheep have a bodyweight approaching that of humans, human scale antibiotic therapy may be applied. Finally, sheep have soft tissue coverage and muscle mass that more closely matches human structures, meaning that overall, large animal models offer the opportunity for a close clinical approximation of the human condition in many ways not possible for smaller animals.

Many large animal models of staphylococcal bone infection have previously been described in the literature. For example, complex device-related infection models have also been described, including osteotomies fixed with IM nailing or external fixation in the goat (Curtis et al., 1995); comparisons of infection after fracture fixation with either primary or secondary bone healing (Rittman and Perren, 1974); comparison of IM nail-related infection secondary to pin tract infection of external fixation (Clasper et al., 2001); measures of neovascularisation after open fractures fixed by an IM nail (Khodaparast et al., 2003). More recently, a large animal model was used in the evaluation of antimicrobial implant surfaces as a prophylaxis against infection in a sheep model of plate osteosynthesis (Schaer et al., 2012; Stewart et al., 2012), resembling other large animal models for prevention of infection using bone cement beads and spacers (Petty et al., 1988; Wenke et al., 2006). The treatment of an established infection has been less frequently covered in the literature, with one study in dogs describing antibiotic-eluting bone cement in a simple bone defect model (Fitzgerald, 1983) and a second canine model of infection associated with a cortical screw (Huneault et al., 2004). A goat model for open wound infection has also been described (Stinner et al., 2012). Extensive staged exchange of chronic infection has not been described for a large or small animal in the literature, to the best of our knowledge. Our staged treatment model significantly advances the field by providing a model for failed treatment, despite both debridement and local and systemic antibiotics. Such a model should prove useful in the robust assessment of novel interventions required to reduce the high failure rate of staged exchange, particularly for MRSA infections.

In developing this model, certain compromises to the clinical situation were made in order to minimise burden to the animals, due to the lack of safety data for some antibiotic regimens in sheep or in order to achieve our stated goal of a failed treatment model enabling investigation of novel interventions. One major compromise was the use of a non-fracture model. A fracture model would be highly desirable since the biomechanics and healing of the fracture are key components and factors in the establishment, treatment and patient outcome for fracture related infection. An artificial mid-diaphyseal osteotomy was created in early trials. However, achieving sufficient stability and performing device removal proved to be a challenge. Performing such procedures in an infected model was deemed high risk and so a decision was made to pursue a non-fracture model. 
This remains a potential area for refinement of the model in future, although the research question involved should clearly mandate the need for a fracture to justify the additional burden and risk to the animals. To improve relatedness to PJI, we could consider an intra-articular entry point for the nail and retain hardware closer to the joint surface. This, however, is likely to result in severe septic arthritis and abscess formation within the joint that, based on experience, is likely to be a high burden for the animal and may compromise the ability to establish a chronic infection.

As mentioned, our overarching study goal was to have a model for failed revision that could serve as a testbed for testing novel interventions. Therefore, a complete cure of infection was not a target per se. Consequently, our guiding principle in antibiotic selection was to administer antibiotics with known safety and acceptable pharmacokinetics that had been proven in sheep. Trimethoprim sulphadoxine was selected as the pathogen-adapted systemic antibiotic for the MRSA group since it has previously been shown to be tolerated by sheep, when used in an MSSA infection model (Clasper et al., 2001). The used MRSA strain was susceptible to this antibiotic, which is licensed for use in sheep (safety and tolerability data available) and is also a suggested oral option for human patients (Zimmerli and Clauss, 2015) after initial IV administration and partnered with rifampicin (Zimmerli et al., 2004).

The limitations of the model included the bacteriological cultures at RSx2 being taken whilst the animals were still under antibiotic treatment. This may have resulted in some false negative culture results. It should be noted that our primary outcome was culture positivity rate at euthanasia, which was determined after a 4 weeks wash-out period with no risk of antibiotic interference in the main primaryoutcome measure. Another potential limitation was that we utilised a dose of bacteria exceeding the likely contamination expected at surgical sites. While it may be possible to achieve infection with a lower inoculum, the $2 \times 10^{7} \mathrm{CFU}$ dose did not result in a systemic infection, but rather resulted in a consistent localised infection tolerated by the sheep and, as such, was deemed adequate for the purposes of our experimental model. We utilised a small piece of collagen fleece to deliver the inoculum, which helped to retain the inoculated bacteria within the deep tissues and reduce the risk of soft tissue infection that might result in wound dehiscence. The collagen may have also protected the inoculum from host defences and ensured access of the bacteria to the implant surface for biofilm formation, both of which are considered to be beneficial for the induction of a persistent localised infection. An additional discussion point is that the sheep displayed early clinical signs of infection and, in human care, this would certainly have led to surgical and/or medical intervention prior to the 8 weeks interventional time point. S. aureus may be more commonly associated with acute rather than chronic infections and may be considered for early intervention with debridement and implant retention. However, in a portion of cases, the first interventions may be inappropriate or insufficient to eradicate the infection, which leads to a chronic mishandled infection, which was the clinical target in mind when developing this model.

In future, this model may be refined in order to verify the suitability of current human guidelines for therapy of chronic ODRI. As examples of the refinements that may be made to more closely resemble human therapy, the duration of systemic antibiotic therapy could be extended, the debridement protocol could be more radical (reaming or jetlavage), repeat debridement could be performed until confirmed pathogen clearance and rifampicin and vancomycin could be added to the treatment regimen, particularly for the MRSA group, which did not respond to the therapy. Rifampicin is a cornerstone of the treatment of device-related bone infection (Trampuz and Zimmerli, 2006; Zimmerli et al., 2004) and the impact of its addition would be instructive. With a view to the novel interventions that may be interesting candidates to be tested in this model, it would be interesting to investigate alternative antibiotics within the cement nail, in a pathogen-adapted manner as was done for the systemic antibiotic therapy. Currently, only gentamicin, vancomycin and clindamycin are commercially available for inclusion in bone cement in the EU, although off-label antibiotic addition, for example with other antibiotics, would be of interest. Non-antibiotic interventions would be particularly of interest, including antimicrobial peptides, immunisation strategies or immune modulation. Other novel means of local antibiotic delivery, such as cement beads or hydrogels, may also be suitable test interventions for future studies to determine if they may enable clearance of infection in this model. The measurement of antibiotic concentrations in the bone, bone marrow or surrounding soft tissue would be of significant value in understanding the success and/or failure of conventional or novel treatment regimens.

\section{Conclusion}

We have successfully developed a clinically relevant large animal model for a two-stage exchange of an IM nail-associated infection. A model for failed treatment is achieved in the case of MRSA infection, despite local and systemic antibiotic therapy. This model is suitable for further studies, particularly those looking into testing new intervention strategies aimed at reducing treatment failure in ODRI. 


\section{Acknowledgements}

This study was supported by funding from AO Trauma as part of the clinical priority programme Bone infection. The funding body had no role in study performance or decision to publish.

Katharina Kluge, Sarah Peters, Markus Wilke, Philipp Zerbe, Philipp Poxleitner, Simon Hackl, Christoph Erichsen, Mario Morgenstern, Iris Keller, Willem-Jan Metsemakers, Barbara Stanic, Julian Fischer, Pamela Furlong, Nicolo Cosmelli, Nora Goudsouzian and Mauro Bluvol, all from AO Research Institute Davos, are acknowledged for their expert assistance in the design, performance and discussion of the present work.

\section{References}

Boxma H, Broekhuizen T, Patka P, Oosting H (1996) Randomised controlled trial of single-dose antibiotic prophylaxis in surgical treatment of closed fractures: the Dutch Trauma Trial. Lancet 347: 11331137.

Brady RA, O’May GA, Leid JG, Prior ML, Costerton JW, Shirtliff ME (2011) Resolution of Staphylococcus aureus biofilm infection using vaccination and antibiotic treatment. Infect Immun 79: 1797-1803.

Calabro L., Lutton C., Din A.F.S.E., Richards R.G., Moriarty T.F. (2013) Animal models of orthopedic implant-related infection. In: Moriarty T., Zaat S., Busscher H. (eds) Biomaterials Associated Infection. Springer, New York, NY: 273-304.

Clasper JC, Stapley SA, Bowley DM, Kenward CE, Taylor V, Watkins PE (2001) Spread of infection, in an animal model, after intramedullary nailing of an infected external fixator pin track. J Orthop Res 19: 155-159.

Curtis MJ, Brown PR, Dick JD, Jinnah RH (1995) Contaminated fractures of the tibia: a comparison of treatment modalities in an animal model. J Orthop Res 13: 286-295.

Fitzgerald RH, Jr. (1983) Experimental osteomyelitis: description of a canine model and the role of depot administration of antibiotics in the prevention and treatment of sepsis. J Bone Joint Surg Am 65: 371-380.

Gatin L, Saleh-Mghir A, Tasse J, Ghout I, Laurent F, Cremieux AC (2014) Ceftaroline-Fosamil efficacy against methicillin-resistant Staphylococcus aureus in a rabbit prosthetic joint infection model. Antimicrob Agents Chemother 58: 6496-6500.

Haleem AA, Berry DJ, Hanssen AD (2004) Mid-term to long-term followup of two-stage reimplantation for infected total knee arthroplasty. Clin Orthop Relat Res 428: 35-39.

Huneault LM, Lussier B, Dubreuil P, Chouinard L, Desevaux C (2004) Prevention and treatment of experimental osteomyelitis in dogs with ciprofloxacin- loaded crosslinked high amylose starch implants. J Orthop Res 22: 1351-1357.

Inzana JA, Schwarz EM, Kates SL, Awad HA (2015) A novel murine model of established Staphylococcal bone infection in the presence of a fracture fixation plate to study therapies utilizing antibiotic-laden spacers after revision surgery. Bone 72: 128-136.

Khodaparast O, Coberly DM, Mathey J, Rohrich RJ, Levin LS, Brown SA (2003) Effect of a transpositional muscle flap on VEGF mRNA expression in a canine fracture model. Plast Reconstr Surg 112: 171-176.

Kilgus DJ, Howe DJ, Strang A (2002) Results of periprosthetic hip and knee infections caused by resistant bacteria. Clin Orthop Relat Res: 116-124.

Kurtz SM, Lau E, Schmier J, Ong KL, Zhao K, Parvizi J (2008) Infection burden for hip and knee arthroplasty in the United States. J Arthroplasty 23: 984-991.

Lichstein P, Gehrke T, Lombardi A, Romano C, Stockley I, Babis G, Bialecki J, Bucsi L, Cai X, Cao L, de Beaubien B, Erhardt J, Goodman S, Jiranek W, Keogh P, Lewallen D, Manner P, Marczynski W, Mason JB, Mulhall K, Paprosky W, Patel P, Piccaluga F, Polkowski G, Pulido L, Stockley I, Suarez J, Thorey F, Tikhilov R, Velazquez JD, Winkler H (2014) Onestage versus two-stage exchange. J Orthop Res 32 Suppl 1: S141-146.

Morandi S, Brasca M, Andrighetto C, Lombardi A, Lodi R (2009) Phenotypic and genotypic characterization of Staphylococcus aureus strains from italian dairy products. Int J Microbiol 2009: 501362.

Morgenstern M, Post V, Erichsen C, Hungerer S, Buhren V, Militz M, Richards G, Moriarty F (2016) Biofilm formation increases treatment failure in Staphylococcus epidermidis device-related osteomyelitis of the lower extremity in human patients. J Orthop Res 11: 1905-1913.

Mortazavi SM, Vegari D, Ho A, Zmistowski B, Parvizi J (2011) Two-stage exchange arthroplasty for infected total knee arthroplasty: predictors of failure. Clin Orthop Relat Res 469: 3049-3054.

Moskowitz JS, Blaisse MR, Samuel RE, Hsu HP, Harris MB, Martin SD, Lee JC, Spector M, Hammond PT (2010) The effectiveness of the controlled release of gentamicin from polyelectrolyte multilayers in the treatment of Staphylococcus aureus infection in a rabbit bone model. Biomaterials 31: 6019-6030.

Osmon DR, Berbari EF, Berendt AR, Lew D, Zimmerli W, Steckelberg JM, Rao N, Hanssen A, Wilson WR, Infectious Diseases Society of America (2013) Diagnosis and management of prosthetic joint infection: clinical practice guidelines by the Infectious Diseases Society of America. Clin Infect Dis 56: 1-10.

Patzakis MJ, Wilkins J (1989) Factors influencing infection rate in open fracture wounds. Clin Orthop Relat Res 243: 36-40.

Pelt CE, Grijalva R, Anderson L, Anderson MB, Erickson J, Peters CL (2014) Two-stage revision TKA is associated with high complication and failure rates. Adv Orthop 2014: 659047. 
Petty W, Spanier S, Shuster JJ (1988) Prevention of infection after total joint replacement. Experiments with a canine model. J Bone Joint Surg Am 70: 536539.

Reizner W, Hunter JG, O'Malley NT, Southgate RD, Schwarz EM, Kates SL (2014) A systematic review of animal models for Staphylococcus aureus osteomyelitis. Eur Cell Mater 27: 196-212.

Rittman WW, Perren S (1974) Cortical bone healing after internal fixation and infection. SpringerVerlag, Berlin Heidelberg New York: 1-76.

Schaer TP, Stewart S, Hsu BB, Klibanov AM (2012) Hydrophobic polycationic coatings that inhibit biofilms and support bone healing during infection. Biomaterials 33: 1245-1254.

Sherrell JC, Fehring TK, Odum S, Hansen E, Zmistowski B, Dennos A, Kalore N, Periprosthetic Infection C (2011) The Chitranjan Ranawat Award: fate of two-stage reimplantation after failed irrigation and debridement for periprosthetic knee infection. Clin Orthop Relat Res 469: 18-25.

Stewart S, Barr S, Engiles J, Hickok NJ, Shapiro IM, Richardson DW, Parvizi J, Schaer TP (2012) Vancomycin-modified implant surface inhibits biofilm formation and supports bone-healing in an infected osteotomy model in sheep: a proof-ofconcept study. J Bone Joint Surg Am 94: 1406-1415.

Stinner DJ, Hsu JR, Wenke JC (2012) Negative pressure wound therapy reduces the effectiveness of traditional local antibiotic depot in a large complex musculoskeletal wound animal model. J Orthop Trauma 26: 512-518.

Strange S, Whitehouse MR, Beswick AD, Board T, Burston A, Burston B, Carroll FE, Dieppe P, Garfield K, Gooberman-Hill R, Jones S, Kunutsor S, Lane A, Lenguerrand E, MacGowan A, Moore A, Noble S, Simon J, Stockley I, Taylor AH, Toms A, Webb J, Whittaker JP, Wilson M, Wylde V, Blom AW (2016) One-stage or two-stage revision surgery for prosthetic hip joint infection--the INFORM trial: a study protocol for a randomised controlled trial. Trials 17: 90.

Sugarman B, Young EJ (1989) Infections associated with prosthetic devices: magnitude of the problem. Infect Dis Clin North Am 3: 187-198.

Trampuz A, Zimmerli W (2006) Diagnosis and treatment of infections associated with fracturefixation devices. Injury 37 Suppl 2: S59-66.

Wasko MK, Borens O (2013) Antibiotic cement nail for the treatment of posttraumatic intramedullary infections of the tibia: midterm results in 10 cases. Injury 44: 1057-1060.

Wasko MK, Kaminski R (2015) Custom-made antibiotic cement nails in orthopaedic trauma: review of outcomes, new approaches, and perspectives. Biomed Res Int 2015: 387186.

Wenke JC, Owens BD, Svoboda SJ, Brooks DE (2006) Effectiveness of commercially-available antibiotic-impregnated implants. J Bone Joint Surg Br 88: 1102-1104.
Zimmerli W (2014) Clinical presentation and treatment of orthopaedic implant-associated infection. J Intern Med 276: 111-119.

Zimmerli W, Clauss M (2015) Periprosthetic joint infection after total hip and knee arthroplasty. In: Bone and Joint Infections, John Wiley \& Sons, Ltd: 131-150.

Zimmerli W, Trampuz A, Ochsner PE (2004) Prosthetic-joint infections. N Engl J Med 351: 16451654.

\section{Discussion with Reviewers}

Volker Alt: A major difference in the study design of this work compared to the human situation is that antibiotics were ceased after RSx2, whereas in the human situation the new implant would be protected from reinfection by (biofilm-killing) antibiotics. Why was this not done in the animal trial?

Authors: Our goal in developing this model was to achieve a model for failed revision. Should we have performed all possible interventions to a maximum, it might be that we could have eradicated the MRSA infection. Interestingly, we could see that a relatively incomplete therapy can effectively treat MSSA infection. We feel that the MRSA model, as described, offers a sound model for a failed revision and it is particularly suitable for testing novel, non-antibiotic interventions. There are, of course, opportunities to improve the treatment efficacy and verify current intervention strategies, although this was not the stated goal of this particular study.

Volker Alt: Results of quantitative bacteriology (Fig. 6) suggested that both systemic (Fig. 6B) and systemic + local antibiotic treatment (Fig. 6D) led to eradication of the infection at the time of RSx2 against MSSA. However, after reinsertion of a nail, only animals treated with systemic and local antibiotics remained infection-free at the time of euthanasia. What is the explanation for this?

Authors: There were several factors at play at the time of RSx2. In all antibiotic-treated groups, there were antibiotic agents in the tissues at the time samples were collected. This would increase the risk of false negative culture results. Also, at RSx2, we only sampled the local tissues with a brush, rather than taking extensive biopsies, which may be associated with a high risk of sampling error. This means that the culture results at RSx2 should be interpreted with caution and we did not consider culture results at RSx2 a primary outcome measure for our model. The critical result, as is also the case for human patients undergoing treatment, is whether the final outcome is successful treatment of the infection or recurrence.

Volker Alt: How would you explain the discrepancy between the high and significant weight loss for the systemic and local group and the complete 
eradication of bacteria at RSx2 and at the time of euthanasia?

Authors: We believe that the primary reason for weight loss in all groups was the initial 8 weeks infection phase, where the infection proceeded unchecked and that animals slowly started to recover once treatment was administered. Having said this, it is true that the systemic and local group had lower weight at RSx2. Our only explanation for this observation was a slow recovery in weight from the infection period.

Volker Alt: Why were only relatively low doses of antibiotics being used in the spacers $(0.5 \mathrm{~g}$ of gentamicin and $2 \mathrm{~g}$ of vancomycin) from commercially available PMMA bone cements, whereas literature recommends (Citak et al., 2014) higher doses of antibiotics in PMMA spacers for infection treatment? Authors: As stated above, our primary goal was to develop a model for failed revision. Using the standard antibiotic dose, rather than high dose, proved to be effective in treating the MSSA infection. The standard dose was obviously ineffective in treating the MRSA infection. Addition of high dose antibiotic cement would improve the treatment protocol and bring us closer to a human standard of care regimen. However, as clearly stated in the manuscript, our goal was to have a model for failed revision and so such a step would fit nicely into a regimen aimed at verifying the value of current human clinical treatment guidelines.

Hani Awad: Based on the literature and your understanding of the mechanisms of osteomyelitis, how would an injury and the ensuing inflammatory and reparative responses influence the infection and treatment?

Authors: Unfortunately, the scientific literature does not provide much data with regards to the specific impact the presence of a fracture may have upon an implant-related bone infection. It is widely accepted in clinical practice that instability across an infected fracture is a risk factor for treatment failure. From this clinical basis, we expected that the likelihood of treatment failure could increase if we were to incorporate an unstable fracture in our sheep model. A stable fracture on the other hand, may not have such a high risk for treatment failure. At the present time, these beliefs have arisen from clinical experience rather than scientific study and, as such, the mechanisms have not been elucidated. However, it may be expected that vascularity in the region of the fracture and, therefore, infiltration of immune cells may be impeded in unstable fractures.

We have studied the mechanisms behind fracturerelated infection in more detail in a mouse model
(Rochford et al., 2016), as mice are more amenable to studying the biological responses to infection than sheep. In that mouse model, we have shown that fracture healing is characterised by elevated interleukin 4 and interferon-gamma gene expression and secretion of these cytokines by bone cells within the healing fracture area. Staphylococcus aureus infection was characterised by a lack of healing, significant osteolysis and a T helper 17 cells (TH17)led bone response that was ineffectual in clearing the infection. Histologically, these fractures had significant infiltration of inflammatory cells and osteolysis at the fracture ends. In our sheep model, with inoculation through a drill hole, we observed radiographic signs of infection and of course our bone biopsies were culture positive, confirming that the sheep had a significant bone infection. As such, many key features of a fracture-related infection were accurately replicated in the model. Should the sheep model in future proceed to a full fracture model, it is reasonable to expect that the local inflammatory environment would be magnified, vascularity may be more significantly compromised and the impact of osteolysis would be greater, although this would need to be confirmed in any such study. It is certain, however, that the fracture would complicate implant removal and add variability in terms of healing due to stability differences or damage to the healing fracture during implant removal.

In summary, the presence of a fracture may increase the risk of treatment failure in this model, especially in an unstable situation. However, this would likely be associated with an increase in burden for the animal and increased variability within groups and so should be weighed against the scientific need for a fracture to answer the research question.

\section{Additional References}

Citak M, Argenson JN, Masri B, Kendoff D, Springer B, Alt V, Baldini A, Cui Q, Deirmengian GK, del Sel H, Harrer MF, Israelite C, Jahoda D, Jutte PC, Levicoff E, Meani E, Motta F, Pena OR, Ranawat AS, Safir O, Squire MW, Taunton MJ, Vogely C, Wellman SS (2014) Spacers. J Orthop Res 32 Suppl 1: S120-129.

Rochford ET, Sabaté Brescó M, Zeiter S, Kluge K, Poulsson A, Ziegler M, Richards RG, O'Mahony L, Moriarty TF (2016) Monitoring immune responses in a mouse model of fracture fixation with and without Staphylococcus aureus osteomyelitis. Bone 83: 82-92.

Editor note: The scientific editor for this paper was Juerg Gasser. 